\title{
Scheduling of Energy Harvesting for MIMO Wireless Powered Communication Networks
}

\author{
Ibrahim Pehlivan and Sinem Coleri Ergen, Senior Member, IEEE
}

\begin{abstract}
Radio frequency (RF) energy harvesting has the potential to provide perpetual energy to the nodes in communication networks. In this paper, we study the optimization problem for the scheduling of the RF energy harvesting to satisfy the energy demands of the links in a wireless powered network containing a multi-antenna hybrid beamforming base station and multi-antenna users: The time is divided into multiple slots, where different beamforming weights are assigned to each slot. Upon formulation of the problem as a non-convex quadratically constrained linear program, we propose a solution method based on alternating minimization algorithm. We demonstrate via simulations that the additional degrees of freedom introduced by the scheduling algorithm can reduce the number of required RF chains in the hybrid beamforming structure for a certain delay performance, resulting in significant cost savings.
\end{abstract}

Index Terms-RF energy harvesting, wireless powered communication network, hybrid beamforming, scheduling.

\section{INTRODUCTION}

The usage of RF energy harvesting is expected to proliferate over alternative energy harvesting technologies, including natural sources, inductive and magnetic resonant coupling, considering the recent advances in the design of highly efficient RF energy harvesting hardware and many advantages including full control on energy transfer, high charging range and small form factor [1]. Although the optimization of energy harvesting and data communication parameters [2]-[7], and scheduling of information transmission [8], have been widely investigated in the literature, the scheduling of RF energy harvesting has been considered only in a limited context.

Wireless powered communication networks (WPCN) are one of the prominent research areas of RF energy harvesting networks. In WPCN, the wireless nodes harvest energy from the base station in the downlink and then transmit data to the base station in the uplink. Up to now, these networks have employed only one slot for the energy transfer from base station to the wireless nodes. The energy harvesting time duration has been optimized for single antenna access point in [5]. On the other hand, the energy beamforming weights have been optimized together with time duration for multi-antenna access point employing digital beamforming, containing a separate RF chain for each antenna, in [2], [6]. However, the high hardware cost, complexity and power consumption of digital beamforming have led to the investigation of lowcost alternatives with less number of RF chains than antennas:

Ibrahim Pehlivan and Sinem Coleri Ergen are with the department of Electrical and Electronics Engineering, Koc University, Istanbul, Turkey, email: ipehlivan, sergen@ku.edu.tr. This work is supported by Scientific and Technological Research Council of Turkey Grant \#117E241. antenna selection and hybrid beamforming [7]. Decreasing the degrees of freedom in one slot by the reduction in the number of RF chains in these low-cost alternative beamformers, or decreasing the number of antennas altogether, is expected to degrade the performance of RF energy harvesting. Similar to information transmission, this can be overcome by including additional degrees of freedom by scheduling, i.e. dividing the time into multiple slots and assigning different energy beamforming weights to each slot. Such an extension has not been investigated before in the literature.

The goal of this paper is to investigate the optimal scheduling of energy harvesting in WPCN, containing a multi-antenna hybrid beamforming base station and multi-antenna users, and demonstrate the impact of scheduling on the performance improvement. To the best of our knowledge, this is the first work to incorporate scheduling of energy harvesting into communication networks.

\section{System Model and Assumptions}

We consider a WPCN containing an access point equipped with $M$ antennas and $N$ users equipped with $R$ antennas. The access point is connected to a power supply with capability of transferring RF energy to the users. Users do not possess any power supply and harvest energy from the RF transmission of the access point. The communication protocol is assumed to employ a half-duplex dynamic time-division multiple access (TDMA), where each TDMA frame is partitioned into $S$ slots of variable duration for the wireless energy transmission of the access point to the users in the downlink, followed by the slots of variable duration allocated for the information transmission of the users to the access point in the uplink using the harvested energy. We assume that the energy requirement of user $n$ is fixed and equal to $E_{n}$ for $n \in[1, N]$ to avoid the complexity in the first step of the study and better illustrate the gain from the scheduling of energy harvesting. We aim to minimize the total duration of energy harvesting to meet these energy requirements.

The output of the access point at time slot $s$ is given by $\mathbf{x}_{\mathbf{s}}=\mathbf{v}_{\mathbf{s}} y_{s}$, where $\mathbf{v}_{\mathbf{s}} \in \mathbb{C}^{\mathbf{M}}$ is energy beamforming vector and $y_{s}$ is the energy carrying signal. Since $y_{s}$ does not carry information, it can be any arbitrary random signal with certain regulations imposed on its power spectral density. We assume that $y_{s}$ is a white process such that $\mathbb{E}\left(\left\|y_{s}\right\|^{2}\right)=1$. The access point is assumed to contain $M$ antennas in hybrid beamforming structure with $L$ RF chains, each RF chain followed by $K=\frac{M}{L}$ RF phase shifters, where $K$ is an integer. This structure is practically used for base station antenna deployment in current 
cellular systems [9]. Let us denote the digital beamforming coefficient of the $l$-th RF chain at time slot $s$ by $v_{s}^{G_{l}}$ and the set of antennas corresponding to the $l$-th RF chain by the phase shifter group $G_{l}=\{(l-1) K+1,(l-1) K+2, \ldots, l K\}$, for $l \in[1, L]$. Then the output of the antennas at time slot $s$ in the same phase shifter group have the same magnitude, i.e. $\mathbf{v}_{\mathbf{s}}(j)=v_{s}^{G_{l}} e^{j \theta_{j}}$, where $\mathbf{v}_{\mathbf{s}}(j)$ is the $j$-th element of vector $\mathbf{v}_{\mathbf{s}}$ and $\theta_{j}$ is phase shift introduced by the $j$-th phase shifter following the corresponding RF chain, thus, $\left|\mathbf{v}_{\mathbf{s}}(j)\right|=\left|v_{s}^{G_{l}}\right|$ for $j \in G_{l}$. The access point is also assumed to transmit at the maximum power level $P_{A}$ such that $\mathbb{E}\left(\mathbf{x}_{\mathbf{s}}^{\mathbf{H}} \mathbf{x}_{\mathbf{s}}\right)=\left\|\mathbf{v}_{\mathbf{s}}\right\|^{2}=\mathbf{v}_{\mathbf{s}}^{\mathbf{H}} \mathbf{v}_{\mathbf{s}} \leq P_{A}$ at time slot $s$.

We assume that the channel gain matrix from the access point to user $n$ is fixed during the harvesting period, equal to $\mathbf{H}_{\mathbf{n}} \in \mathbb{C}^{\mathbf{M} \times \mathbf{R}}$ for $n \in[1, N]$ and perfectly known by the access point. This block fading channel assumption is commonly used in previous optimization problems formulated for WPCN [2], [5]-[7]. The energy harvested by user $n$ at time slot $s$ is then given by $\psi\left\|\mathbf{H}_{\mathbf{n}}{ }^{\mathbf{H}} \mathbf{v}_{\mathbf{s}}\right\|^{2} t_{s}=\psi \mathbf{v}_{\mathbf{s}}^{\mathbf{H}} \mathbf{H}_{\mathbf{n}} \mathbf{H}_{\mathbf{n}}{ }^{\mathbf{H}} \mathbf{v}_{\mathbf{s}} t_{s}=\psi \mathbf{v}_{\mathbf{s}}^{\mathbf{H}} \mathbf{H}_{\mathbf{n}} \mathbf{v}_{\mathbf{s}} t_{s}$, where $\mathbf{H}_{\mathbf{n}}$ is defined as $\mathbf{H}_{\mathbf{n}}:=\mathbf{H}_{\mathbf{n}} \mathbf{H}_{\mathbf{n}} \overline{\mathbf{H} \in \mathbb{C}} \mathbf{M} \times \mathbf{M}, t_{s}$ is the duration of the $s$-th time slot, and $\overline{0<\psi} \leq 1$ is the energy harvesting efficiency [2]-[7].

\section{Optimization Problem Formulation}

The optimization problem aims to minimize the total energy harvesting time from the access point to the users while satisfying the energy requirement of the users, the maximum transmit power and hybrid beamforming constraints of the wireless communication system.

$$
\begin{aligned}
& \text { minimize } \\
& \sum_{s=1}^{S} t_{s} \\
& \text { subject to } \\
& \sum_{s=1}^{S} \psi \mathbf{w}_{\mathbf{s}}^{\mathbf{H}} \mathbf{H}_{\mathbf{n}} \mathbf{w}_{\mathbf{s}} \geq E_{n} \quad n \in[1, N] \\
& \mathbf{w}_{\mathbf{s}}^{\mathbf{H}} \mathbf{w}_{\mathbf{s}} \leq P_{A} t_{s} \quad s \in[1, S] \\
& \left|\mathbf{w}_{\mathbf{s}}(j)\right|=\left|\mathbf{w}_{\mathbf{s}}(i)\right| \quad i, j \in G_{l}, \\
& l \in[1, L], \quad s \in[1, S]
\end{aligned}
$$

variables

$$
t_{s} \in \mathbb{R}_{+}, \mathbf{w}_{\mathbf{s}} \in \mathbb{C}^{M}, \quad s \in[1, S]
$$

The variables of the optimization problem are $t_{s}$, the duration of the $s$-th time slot, and $\mathbf{w}_{\mathbf{s}}$, the time weighted energy beamforming vector of the $s$-th time slot, defined as $\mathbf{w}_{\mathbf{s}}:=\mathbf{v}_{\mathbf{s}} \sqrt{t_{s}}$ for $t_{s}>0$ and $s \in[1, S]$. The $\mathbf{w}_{\mathbf{s}}$ definition allows the removal of the coupling of the variables $t_{s}$ and $\mathbf{v}_{\mathbf{s}}$ at the harvested energy of the users.

Equation (1b) represents the energy requirement of the users, where the required energy of each user needs to be satisfied by the total energy transferred from the base station. Equation (1c) gives the maximum transmit power constraint of the base station. Equation (1d) states the limitation introduced by the hybrid beamforming structure.
This optimization problem is a non-convex quadratically constrained linear program (QCLP). Non-convexity stems from the constraints in Equations (1b) and (1d). Therefore, we will employ approximation methods next.

\section{Solution Method}

We will examine the usage of semidefinite relaxation and alternating minimization algorithms for the efficient approximation of the non-convex QCLP problem (1).

\section{A. Semidefinite relaxation $(S D R)$}

SDR has been proposed as an efficient approximation technique for problems with non-convex quadratic constraints. In SDR, the non-convex QCLP problem is reformulated by using equivalent positive semidefinite (PSD) matrices and additional rank constraints. Then the solution methodology is composed of first solving the resulting semidefinite programming from the relaxation of the rank constraint and then using rank reduction methods [10].

The reformulation of the non-convex QCLP problem (1) is given by

minimize

$$
\sum_{s=1}^{S} t_{s}
$$

subject to

$$
\begin{aligned}
& \sum_{s=1}^{S} \psi \operatorname{Tr}\left(\mathbf{H}_{\mathbf{n}} \mathbf{W}_{\mathbf{s}}\right) \geq E_{n} \quad n \in[1, N] \\
& \operatorname{Tr}\left(\mathbf{W}_{\mathbf{s}}\right) \leq P_{A} t_{s} \quad s \in[1, S] \\
& \mathbf{W}_{\mathbf{s}}(j, j)=\mathbf{W}_{\mathbf{s}}(i, i) \quad i, j \in G_{l}, \\
& \quad l \in[1, L], \quad s \in[1, S] \\
& \operatorname{rank}\left(\mathbf{W}_{\mathbf{s}}\right)=1 \quad s \in[1, S] \\
& \mathbf{W}_{\mathbf{s}} \geq 0 \quad s \in[1, S]
\end{aligned}
$$

variables

$$
t_{s} \in \mathbb{R}_{+}, \mathbf{W}_{\mathbf{s}} \in \mathbb{C}^{M \times M}, \quad s \in[1, S]
$$

The variables of the optimization problem are $t_{s}$ and $\mathbf{W}_{\mathbf{s}}$, defined as the time weighted beamforming matrix at time slot $s$, equal to $\mathbf{W}_{\mathbf{s}}:=\mathbf{w}_{\mathbf{s}} \mathbf{w}_{\mathbf{s}}^{\mathbf{H}}$. Equation (2b) is equivalent to Equation (1b), based on the fact that $\mathbf{w}_{\mathbf{s}}^{\mathbf{H}} \mathbf{H}_{\mathbf{n}} \mathbf{w}_{\mathbf{s}}=\operatorname{Tr}\left(\mathbf{w}_{\mathbf{s}}^{\mathbf{H}} \mathbf{H}_{\mathbf{n}} \mathbf{w}_{\mathbf{s}}\right)=$ $\operatorname{Tr}\left(\mathbf{H}_{\mathbf{n}} \mathbf{w}_{\mathbf{s}} \mathbf{w}_{\mathbf{s}}^{\mathbf{H}}\right)=\operatorname{Tr}\left(\mathbf{H}_{\mathbf{n}} \mathbf{W}_{\mathbf{s}}\right)$. Similarly, Equations (2c) and (2d) are equivalent to Equations (1c) and (1d), respectively. Additional constraints in Equations (2e) and (2f) are included to enforce that $\mathbf{W}_{\mathbf{s}}$ is rank 1 and positive semidefinite, respectively. Problem (1) is equivalent to problem (2) [10].

By relaxing the only non-convex constraint, rank constraint in Equation (2e), we can obtain semidefinite relaxation of our problem. Relaxed problem is convex and can easily be solved in a numerically reliable and efficient manner by readily available software packages [11]. However this relaxation bound for scheduled, $S>1$, and non-scheduled, $S=1$, cases are identical as proven in the next lemma.

Lemma 1. SDR bound obtained by the relaxation of the rank constraint in the optimization problem (2) is independent of the number of available time slots, $S \geq 1$, for scheduling. 
Proof. Let us denote the relaxed version of the optimization problem (2) with $S$ time slots as $\boldsymbol{\Pi}_{\mathbf{S}}$, its feasible solution as $\mathbf{X}_{\mathbf{S}}=\left\{\left(\mathbf{W}_{\mathbf{s}}\right)_{s=1}^{S},\left(t_{s}\right)_{s=1}^{S}\right\}$, where $\left(\mathbf{W}_{\mathbf{s}}\right)_{s=1}^{S}$ denotes the collection of $S$ time weighted beamforming matrices $\left(\mathbf{W}_{\mathbf{1}}, \mathbf{W}_{\mathbf{2}}, \ldots, \mathbf{W}_{\mathbf{S}}\right)$ and $\left(t_{s}\right)_{s=1}^{S}$ represents the collection of $S$ time slot durations, $\left(t_{1}, t_{2}, \ldots, t_{S}\right)$, and its objective function as $\mathbf{f}_{\mathbf{S}}\left(\mathbf{X}_{\mathbf{S}}\right)$. We prove the lemma by demonstrating that an optimal solution $\mathbf{X}_{\mathbf{1}}$ of the problem $\boldsymbol{\Pi}_{\mathbf{1}}$ can be constructed from an optimal solution $\mathbf{X}_{\mathbf{S}}$ of the problem $\boldsymbol{\Pi}_{\mathbf{S}}$ with the same value of the objective function, and vice versa.

Let $\mathbf{X}_{\mathbf{S}}=\left\{\left(\mathbf{W}_{\mathbf{s}}\right)_{s=1}^{S},\left(t_{s}\right)_{s=1}^{S}\right\}$ be a feasible solution to the problem $\Pi_{\mathbf{S}}$. Due to linearity of trace function, $\mathbf{X}_{\mathbf{1}}=$ $\left\{\left(\sum_{\mathbf{s = 1}}^{\mathbf{S}} \mathbf{W}_{\mathbf{s}}\right),\left(\sum_{s=1}^{S} t_{s}\right)\right\}$ is a feasible solution of the problem $\Pi_{1}$ with the same optimal value. Similarly, given a feasible solution $\mathbf{X}_{\mathbf{1}}$ of the problem $\boldsymbol{\Pi}_{\mathbf{1}}, \mathbf{X}_{\mathbf{S}}=\left\{\left(\mathbf{W}_{\mathbf{1}} / S\right)_{s=1}^{S},\left(t_{1} / S\right)_{s=1}^{S}\right\}$ is a feasible solution of the problem $\Pi_{\mathbf{S}}$ with the same optimal value. Since a feasible solution with the same objective value to one problem can be constructed from the other problem, then by contradiction it can be proven that an optimal solution to one problem can be constructed from the other problem. Since the result is valid for any $S$, SDR bound is independent of the number of available time slots.

Therefore, there is no point in continuing with SDR rank reduction methods. However, the solution of SDR is a good lower bound for the total energy harvesting time.

\section{B. Alternating Minimization (AM) Algorithm}

Alternating minimization (AM) algorithm is based on reformulating the rank constraint as an equivalent non-convex constraint and iteratively solving the convex optimization problem obtained by moving rank constraint to the objective function as a penalty function. The convergence of the objective function of the optimization problem by this iterative algorithm has been proved in [7].

The rank constraint given in Equation (2e) is first replaced by $\operatorname{Tr}\left(\mathbf{W}_{\mathbf{s}}\right)^{2}-\operatorname{Tr}\left(\mathbf{W}_{\mathbf{s}}{ }^{2}\right) \leq 0, s \in[1, S]$, based on the fact that for every non-zero hermitian positive semidefinite matrix $W, \operatorname{Tr}\left(W^{2}\right) \leq \operatorname{Tr}(W)^{2}$; and $\operatorname{Tr}\left(W^{2}\right)=\operatorname{Tr}(W)^{2}$ if and only if $\operatorname{rank}(\mathrm{W})=1$ [7]. This new constraint is non-convex but can be moved to the objective function as a penalty by including the sum of the amount of the violation of the constraint at each time slot $s$, i.e. $\sum_{s=1}^{S} \xi_{s}\left(\operatorname{Tr}\left(\mathbf{W}_{\mathbf{s}}\right) \operatorname{Tr}\left(\mathbf{W}_{\mathbf{s}}\right)-\operatorname{Tr}\left(\mathbf{W}_{\mathbf{s}} \mathbf{W}_{\mathbf{s}}\right)\right)$, where $\xi_{s}$ is the weight of the violation at time slot $s$. Resultant problem can be solved iteratively by AM algorithm. In the $q$-th iteration of the algorithm, the following problem is solved:

$$
\begin{aligned}
\min & \sum_{s=1}^{S} t_{s} \\
& +\sum_{s=1}^{S} \xi_{s}\left(\operatorname{Tr}\left(\mathbf{W}_{\mathbf{s}}{ }^{(q-1)}\right) \operatorname{Tr}\left(\mathbf{W}_{\mathbf{s}}\right)-\operatorname{Tr}\left(\mathbf{W}_{\mathbf{s}}{ }^{(q-1)} \mathbf{W}_{\mathbf{s}}\right)\right)
\end{aligned}
$$

s.t. $\quad(2 b, 2 c, 2 d, 2 f)$

where $\mathbf{W}_{\mathbf{s}}^{(q-1)}$ is the optimal solution for $s \in[1, S]$ at the $(q-1)$-th step of the algorithm.

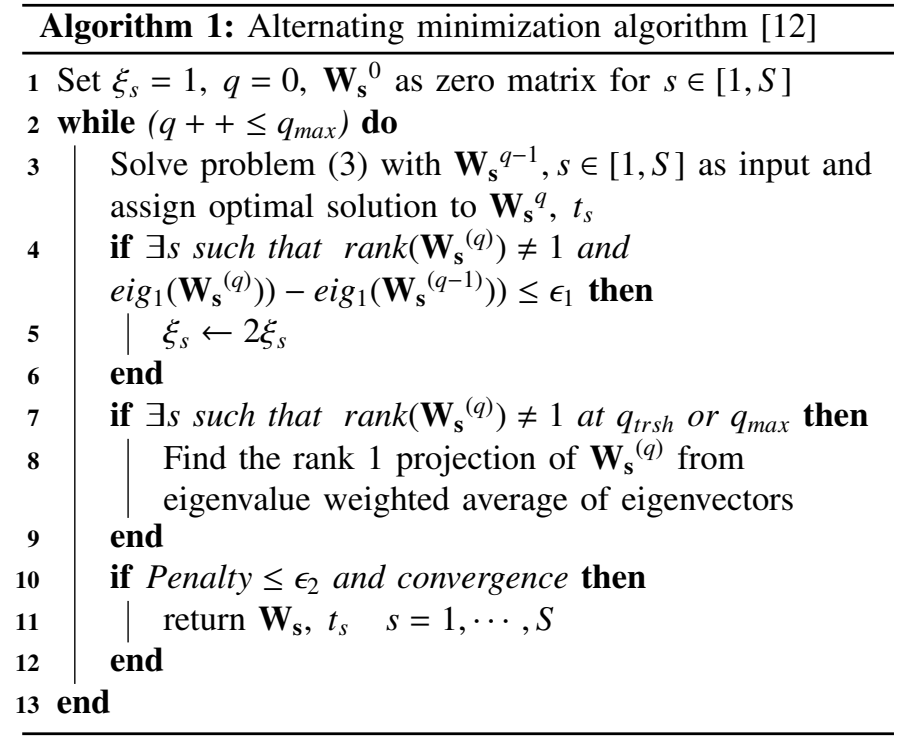

Algorithm (1) provides the details of the implementation of the AM algorithm. $q_{\max }$ is the maximum number of iterations, $q_{t r s h}$ is the iteration threshold where algorithm projects the solution matrices to rank 1 matrices, $e i g_{1}$ is the function which gives the principal eigenvalue of the input matrix, $\epsilon_{1}$ and $\epsilon_{2}$ are desired accuracies, and penalty is the unweighted cost of the constraint violation. Algorithm starts with the initialization (Line 1). Then, at the $q$-th iteration, the optimization problem (3) is solved and corresponding optimal solution is recorded (Line 3). Then the violation weight of the non-rank 1 matrix whose principal eigenvalue is not improved within the desired range $\epsilon_{1}$ is doubled to increase its priority (Lines $4-6$ ). The solution is guaranteed to have rank 1 solution by rank projection at $q_{t r s h}$ and $q_{\max }$ (Lines $7-9$ ). Unlike previous implementations of AM algorithm, this algorithm continues searching around the solution after projection through intelligent configuration of the penalty coefficients and uses weighted sum of eigenvectors instead of primal eigenvector as a solution, after rescaling its elements to satisfy Equation (1d).

\section{Performance analysis}

The goal of this section is to demonstrate the performance gain achieved by the scheduling of energy harvesting for different network topology and hybrid beamforming architectures. The solution of the optimization problem (1) obtained by the AM algorithm is denoted by scheduled for $S=5$ and nonscheduled for $S=1$. To demonstrate the closeness of the proposed algorithm to the optimal solution, the semidefinite programming problem obtained by the removal of the rank constraint in Equation (2e) from Equations (2) is included and denoted by SDR bound.

Simulation results are obtained in the convex programming environment CVX in MATLAB [11], based on 120 independent random network topologies, where the users are distributed randomly on a sphere of radius $4 \mathrm{~m}$. Base station contains 16 antennas with maximum allowed power, $P_{A}, 40$ $\mathrm{dBm}$, complying with FCC base station regulations. The users 


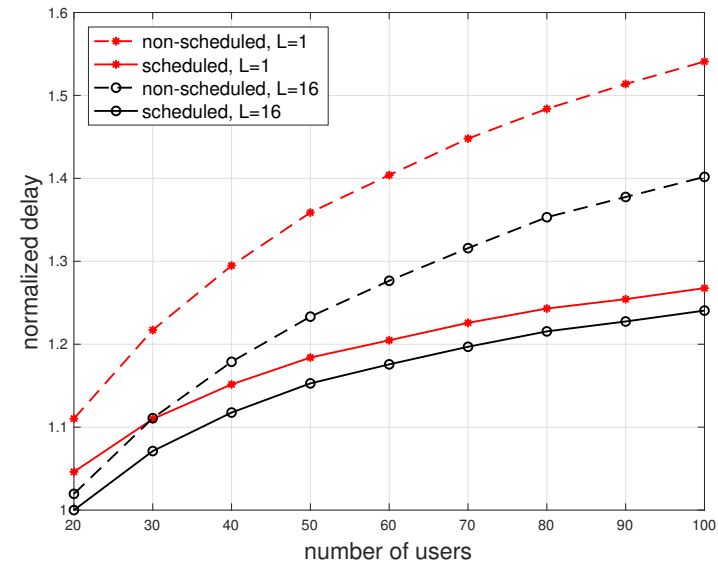

Figure 1. Normalized energy harvesting time for different number of users

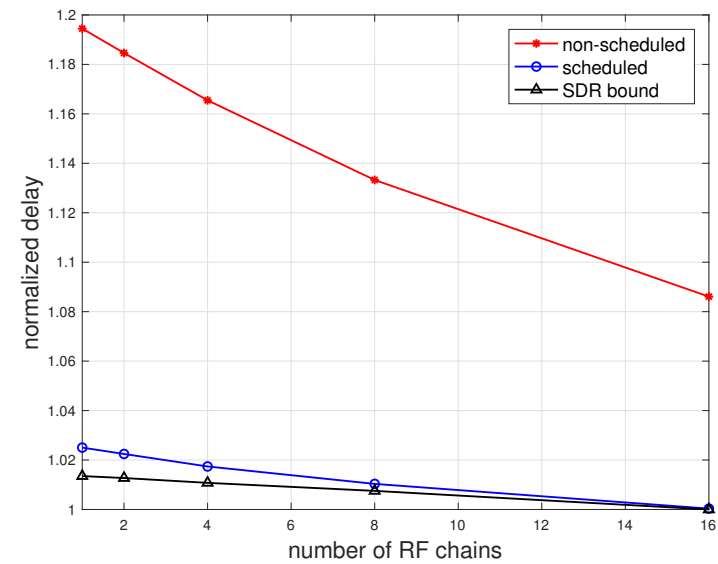

Figure 2. Normalized energy harvesting time in a 60-node network for different number of RF chains

are equipped with 16 antennas. The antenna configuration is cross-shaped antenna array with antenna spacing of $\lambda / 4$, where $\lambda$ is the wavelength of the carrier frequency. The attenuation of the links is determined by using Rayleigh fading with scale parameter set to the mean path loss value calculated by $P L(d)=P L\left(d_{0}\right)+10 \alpha \log \left(d / d_{0}\right)+Z$, where $d$ is the distance between the user and base station, $P L(d)$ is the path loss at distance $d$ in decibels, $P L\left(d_{0}\right)$ is the path loss at reference distance $d_{0}=1 \mathrm{~m}, \alpha$ is the path loss exponent and $Z$ is a Gaussian random variable with zero mean and standard deviation $\sigma_{z}$. The channel parameters are $P L\left(d_{0}\right)=61.39 \mathrm{~dB}$, $\alpha=1.2$ and $\sigma_{z}=1.7 \mathrm{~dB}$ at $28 \mathrm{GHz}$ frequency band for line of sight (LOS) transmission [13]. The required energy for each user $n \in[1, N]$ is $E_{n}=10^{-6} \mathrm{~J}$ and the energy harvesting efficiency is assumed to be $\psi=0.2$.

Fig. 1 shows the normalized energy harvesting time for different number of users. Scheduled beamforming with low $\mathrm{RF}$ chain number $L=1$ is observed to outperform the nonscheduled hybrid beamforming with high RF chain number $L=16$ for more than 30 users. The gain achieved by the incorporation of energy harvesting scheduling increases as the number of users increases. This is mainly due to better exploitation of additional degrees of freedom introduced by scheduling in larger networks.
Fig. 2 shows the normalized energy harvesting time in a 60node network for different numbers of RF chains. Scheduling is observed to decrease the energy harvesting time by more than $\% 8$. The gain by scheduling significantly increases as the number of RF chains decreases, due to higher compensation of the limited number of degrees of freedom at small number of RF chains by scheduling.

\section{Conclusion}

In this paper, we have demonstrated the performance gain by the optimal scheduling of RF energy harvesting in a wireless powered communication network containing a multi-antenna hybrid beamforming base station and multi-antenna users. We observe that the number of required RF chains in the hybrid beamforming structure for a certain delay performance decreases by the use of energy harvesting scheduling. Moreover, the performance gain in energy harvesting time increases as the network size increases and number of RF chains decreases.

\section{REFERENCES}

[1] L. Xie, Y. Shi, Y. T. Hou, and A. Lou, "Wireless power transfer and applications to sensor networks," IEEE Wireless Communications, vol. 20, pp. 140-145, August 2013.

[2] J. Xu, L. Liu, and R. Zhang, "Multiuser miso beamforming for simultaneous wireless information and power transfer," IEEE Transactions on Signal Processing, vol. 62, pp. 4798-4810, September 2014.

[3] J. Tang, D. K. C. So, A. Shojaeifard, K. K. Wong, and J. Wen, "Joint antenna selection and spatial switching for energy efficient mimo swipt system," IEEE Transactions on Wireless Communications, vol. 16, pp. 4754-4769, July 2017.

[4] R. Morsi, D. S. Michalopoulos, and R. Schober, "Multiuser scheduling schemes for simultaneous wireless information and power transfer over fading channels," IEEE Transactions on Wireless Communications, vol. 14, pp. 1967-1982, April 2015.

[5] H. Ju and R. Zhang, "Throughput maximization in wireless powered communication networks," IEEE Transactions on Wireless Communications, vol. 13, pp. 418-428, January 2014.

[6] L. Liu, R. Zhang, and K. C. Chua, "Multi-antenna wireless powered communication with energy beamforming," IEEE Transactions on Communications, vol. 62, pp. 4349-4361, December 2014.

[7] O. T. Demir and T. E. Tuncer, "Antenna selection and hybrid beamforming for simultaneous wireless information and power transfer in multi-group multicasting systems," IEEE Transactions on Wireless Communications, vol. 15, pp. 6948-6962, October 2016.

[8] Y. Sadi and S. C. Ergen, "Minimum length scheduling with packet traffic demands in wireless networks," IEEE Transactions on Wireless Communications, vol. 13, pp. 3738-3751, July 2014.

[9] S. Han, I. Chih-Lin, Z. Xu, and C. Rowell, "Large-scale antenna systems with hybrid analog and digital beamforming for millimeter wave $5 \mathrm{~g}$," IEEE Communications Magazine, vol. 53, pp. 186-194, January 2015.

[10] Z. q. Luo, W. k. Ma, A. M. c. So, Y. Ye, and S. Zhang, "Semidefinite relaxation of quadratic optimization problems," IEEE Signal Processing Magazine, vol. 27, pp. 20-34, May 2010.

[11] M. Grant and S. Boyd, "CVX: Matlab software for disciplined convex programming, version 2.1," Mar. 2014.

[12] O. T. Demir and T. E. Tuncer, "Antenna selection and hybrid beamforming for simultaneous wireless information and power transfer in multi-group multicasting systems," IEEE Transactions on Wireless Communications, vol. 15, pp. 6948-6962, Oct 2016.

[13] S. Deng, M. K. Samimi, and T. S. Rappaport, " $28 \mathrm{ghz}$ and 73 ghz millimeter-wave indoor propagation measurements and path loss models," in 2015 IEEE International Conference on Communication Workshop (ICCW), pp. 1244-1250, June 2015. 